\title{
Plasma fibrinogen may predict persistent infection before reimplantation in two- stage exchange arthroplasty for periprosthetic hip infection
}

\author{
Chi Xut, Peng-Fei Qu+, Wei Chai, Rui Li and Ji-Ying Chen ${ }^{*}$
}

\begin{abstract}
Background: The diagnosis of persistent infection before reimplantation in two-stage exchange arthroplasty for periprosthetic joint infection (PJI) remains challenging. Currently, several studies suggested coagulation-related markers, such as D-dimer and fibrinogen, may be promising in diagnose of PJI. The purpose of the study was to investigate the predictive values of plasma D-dimer and fibrinogen for assessment of persistent infection before reimplantation hip arthroplasty.

Methods: We retrospectively reviewed 129 hips that treated with two-stage exchange arthroplasty for PJI from 2012 to 2016 in our institution. The persistent infection before reimplantation was based on a modified Musculoskeletal Infection Society (MSIS) criteria. After exclusion, 102 hips were included in the final analysis. Receiver operating characteristic (ROC) curves were generated to determine the prognostic value of plasma D-dimer and fibrinogen in predicting persistent infection before reimplantation.

Results: The area the under ROC curves (AUC) for fibrinogen (0.773; 95\% confidential interval [Cl], 0.569-0.905) was significantly higher than that of D-dimer $(0.565 ; 95 \% \mathrm{Cl}, 0.329-0.777)$. With the calculated threshold of fibrinogen set at 3 . $61 \mathrm{~g} / \mathrm{L}$, the sensitivity, specificity, positive predictive value (PPV), and negative predictive value (NPV) was 87.5\%, 62.8\%, 16. $7 \%$, and $98.3 \%$, respectively. With the threshold value of D-dimer set at $0.82 \mu \mathrm{g} / \mathrm{mL}$, the sensitivity, specificity, PPV, and NPV was $83.3 \%, 41.9 \%, 21.7 \%$, and $92.9 \%$, respectively.

Conclusions: In conclusion, the current study reveals that the plasma fibrinogen may be a promising biomarker in predicting persistent infection before reimplantation. Further prospective studies with larger cohorts are needed to validate predictive values and optimal thresholds of coagulation-related markers.
\end{abstract}

Keywords: Periprosthetic joint infection, Two-stage exchange arthroplasty, D-dimer, Fibrinogen

\section{Introduction}

The management of periprosthetic joint infection (PJI) following total joint arthroplasty remains challenging. Although two-stage exchange arthroplasty has been the preferred treatment for chronic PJI in North America, the reported rate of re-infection is even up to $30 \%$ [1-3]. The rationale behind this unacceptable outcome could

\footnotetext{
*Correspondence: jiying_chen301@163.com

${ }^{\dagger} \mathrm{Chi} \mathrm{Xu}$ and Peng-Fei Qu contributed equally to this work and are considered co-first authors.

Department of Orthopaedic Surgery, General Hospital of People's Liberation Army, No. 28 Fuxing Road, Haidian District, Beijing 100853, China
}

be the difficulty in exclusion of persistent infection before second-stage reimplantation [4-8].

Numerous attempts have been made to determine the eradication of infection before reimplantation. However, prior studies have suggested limited predictive values of the widely used biomarkers for diagnosis of PJI, including serum erythrocyte sedimentation rate (ESR) and C-reactive protein (CRP) $[8,9]$, synovial white blood cell (WBC) count and percentage of polymorphonuclear cells (PMN\%) [7], aspiration culture [10], and histology analysis $[11,12]$. Additionally, aspiration performed on hips with

(c) The Author(s). 2019 Open Access This article is distributed under the terms of the Creative Commons Attribution 4.0 International License (http://creativecommons.org/licenses/by/4.0/), which permits unrestricted use, distribution, and 
the antibiotic spacer can be hard with a high risk of "dry tap" despite the aid of fluoroscopic guidance $[7,13]$.

The literature has indicated the existence of closed correlations between the coagulation cascade and infection/inflammatory mechanisms [14, 15]. Recently, several studies have suggested the promising value of coagulation-related biomarkers, such as D-dimer and fibrinogen in the diagnosis of PJI $[16,17]$. However, its ability to identify persistent infection at the time of reimplantation remains unknown.

The purpose of the present study was to investigate (1) the diagnostic values of plasma D-dimer and fibrinogen for assessment of persistent infection before reimplantation hip arthroplasty and (2) the best thresholds of D-dimer and fibrinogen that are predictive of persistent PJI.

\section{Methods}

After the Institutional Review Board approval, we retrospectively reviewed all consecutive PJIs that were treated with two-stage exchange arthroplasty for periprosthetic hip infection from 2012 to 2016 in our institution. Of them, 129 two-stage exchanges (129 patients) were reimplanted without any surgeries between the first and second stage. The diagnosis of PJI was according to the Musculoskeletal Infection Society (MSIS) criteria [18].
Patients with a history of venous thrombus embolism (VTE), malignancy, and liver diseases and patients with missing critical data were excluded. As hip aspirations were not routinely performed prior to the second-stage reimplantation in our institution, the diagnosis of persistent infection was based on a modified MSIS criteria [7, 18]: the presence of a sinus tract communicating with the joint at surgery or two positive intraoperative periprosthetic cultures with the same organism or fulfill all the following three minor criteria including (1) ESR $>30 \mathrm{~mm} / \mathrm{h}$ and CRP $>10 \mathrm{mg} / \mathrm{L}$, (2) a single positive intraoperative periprosthetic tissue culture, and (3) a positive histologic analysis of periprosthetic tissue [>5 neutrophils per high power field]. Seven cases that satisfied two of the minor criteria were considered as suspected infection and were also excluded. Finally, 8 hips (group 1) and 94 controls, including 40 hips with one of the minor criteria (group 2) and 54 cases without any minor criteria (group 3), were included in the final analysis (Fig. 1).

Plasma fibrinogen and D-dimer values were routinely obtained before reimplantation after admission. Fibrinogen and D-dimer levels were measured by STA-R Evolution $^{\circ}$ analyzer (Stago Diagnostica, Asnieres, France) and expressed in gram per liter $(\mathrm{g} / \mathrm{L})$ and microgram per milliliter $(\mu \mathrm{g} / \mathrm{mL})$, respectively. Medical records were reviewed

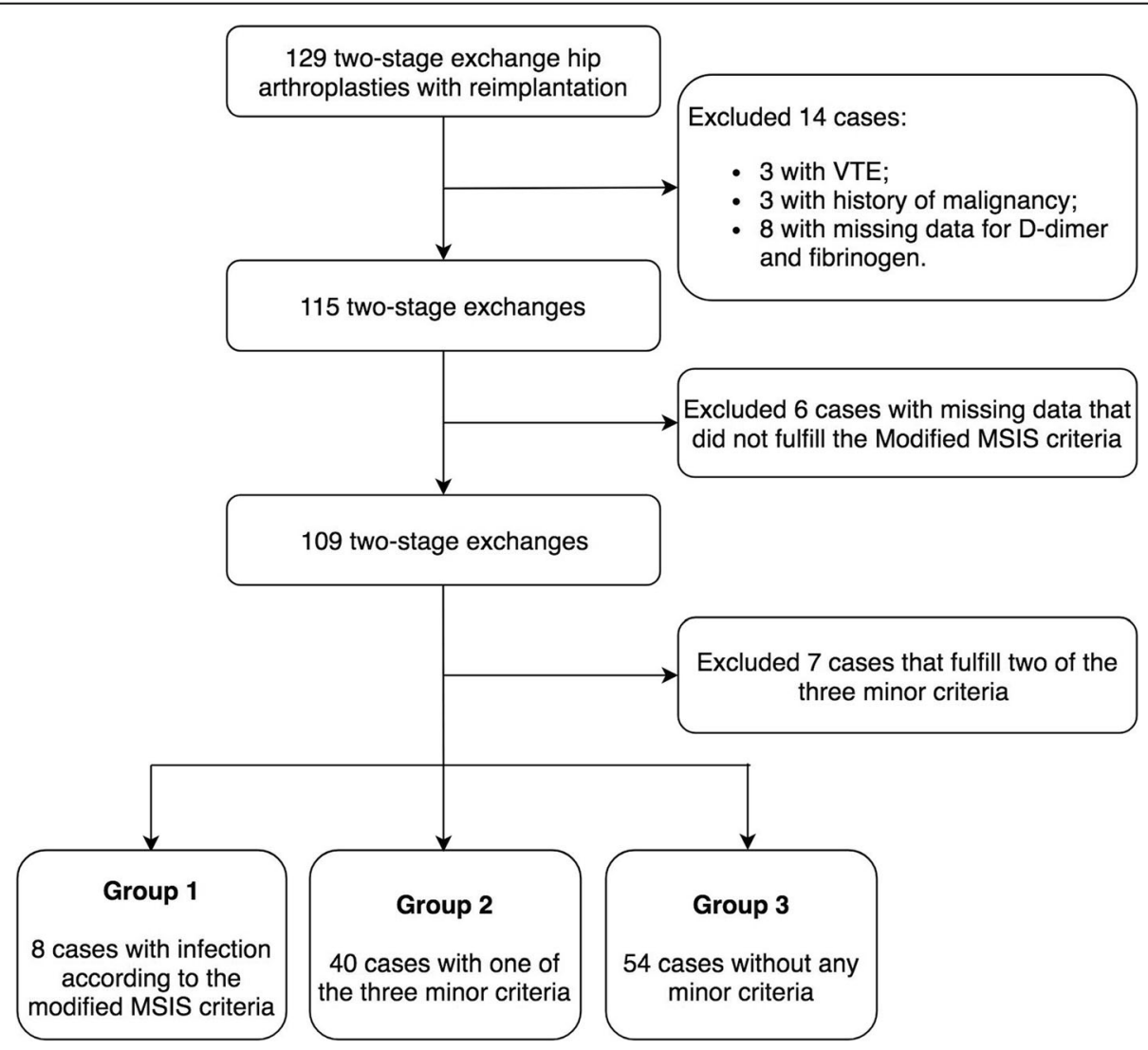

Fig. 1 Flowchart 
manually to retrieve pertinent information including age, gender, body mass index (BMI), serum CRP and ESR values, intraoperative cultures, and histologic results.

An institutional standard protocol of two-stage exchange arthroplasty was performed. During the first-stage resection arthroplasty, all implanted components were removed followed by extensive debridement and irrigation. An articular antibiotic-loaded cement spacer, containing 6-10 g of vancomycin and 2-4 g of meropenem per $40 \mathrm{~g}$ bone cement (Heraeus Medical GmbH, Wehrheim/Ts., Germany), was then inserted. The combination of vancomycin and meropenem in the bone cement was utilized in accordance with our institutional infection control department, which explained that more than $90 \%$ of the organisms isolated from patients with PJI were sensitive to one or both antibiotics. Following resection arthroplasty, 4 weeks of prophylactic anticoagulation (Rivaroxaban, $5 \mathrm{mg}$ PO q 24h) and 6-8 weeks of systemic antibiotic therapy were prescribed. The selection of antibiotic application was based on culture sensitivity reports and institutional guidelines infectious specialists' consultation. The timing of reimplantation was based on the following criteria: no clinical signs of infection, a well-healed surgical wound, and gradually decreased of ESR and CRP. Fibrinogen and D-dimer values were not used to make decisions. All patients had a period of at least 2-week antibiotic holiday before second-stage reimplantation. During the second-stage surgery, antibiotic-loaded cement spacer was removed. Four to six tissue samples for culture and three to five samples for histology analysis were taken from the periprosthetic membrane and other periprosthetic tissues in which infection was suspected. Then, the prostheses were implanted followed by re-debridement and irrigation. Parenteral antibiotics were given postoperatively until the intraoperative cultures were negative findings.

\section{Statistical analysis}

All of the statistical analyses were performed with the statistical software packages $\mathrm{R}$ (http://www.R-project.org, The $\mathrm{R}$ Foundation). Categorical variables were presented as frequencies and percentages and continuous variables as means and standard deviation or median and range. The differences between groups were compared with the use of the Mann-Whitney test for continuous variables and the
Fisher's exact test for categorical variables. Patient characteristics were shown in Table 1 . There was no significant difference in age, gender, and BMI between groups.

Receiver operating characteristic (ROC) curves were generated using bootstrap resampling (times $=500$ ) [19] to determine the performance of plasma fibrinogen and $\mathrm{D}$-dimer in predicting persistent infection at the time of reimplantation. The area under the ROC curve (AUC) with 95\% CI was used as a measure of diagnostic accuracy. AUCs were compared by using the DeLong method [20]. A $p$ value of 0.05 was considered significant.

\section{Sensitivity analysis}

For no gold standard criteria for persistent infection at reimplantation, we performed a set of sensitivity analyses to further validate our results. We reevaluated the diagnostic value of plasma fibrinogen and D-dimer by only using group 1 as infection cases and group 3 (54 cases without any minor criteria) as controls to validate results. Forty hips with one of the minor criteria in group 2 were excluded in the sensitivity analysis. Then, the ROC curves were made and AUCs were compared according to the aforementioned methods.

\section{Results}

The plasma fibrinogen level was significantly higher in the patients with persistent infection ( $p=0.032$; Fig. 2$)$; the median fibrinogen level was $4.3 \mathrm{~g} / \mathrm{L}$ (range $3.0-6.9 \mathrm{~g} / \mathrm{L}$ ) in group 1 compared with $3.3 \mathrm{~g} / \mathrm{L}$ (range $2.2-6.6 \mathrm{~g} / \mathrm{L}$ ) in group 2 and $3.4 \mathrm{~g} / \mathrm{L}$ (range $2.1-6.7 \mathrm{~g} / \mathrm{L}$ ) in group 3 . There was no difference in D-dimer level among groups $(p=$ 0.745; Fig. 2); the median D-dimer level of groups 1,2 , and 3 was $1.6 \mu \mathrm{g} / \mathrm{mL}$ (range $0.3-3.2 \mu \mathrm{g} / \mathrm{mL}$ ), $1.1 \mu \mathrm{g} / \mathrm{mL}$ (range $0.3-3.5 \mu \mathrm{g} / \mathrm{mL}$ ), and $1.2 \mu \mathrm{g} / \mathrm{mL}$ (range $0.4-3.7 \mu \mathrm{g}$ / $\mathrm{mL})$, respectively.

Using the modified MSIS criteria indicating the persistent infection at the second-stage reimplantation, the AUC for fibrinogen (0.773; 95\% CI, 0.569-0.905) was significantly higher than that of D-dimer $(0.565$; 95\% CI, 0.329-0.777) (Fig. 3). With the calculated threshold of fibrinogen set at $3.61 \mathrm{~g} / \mathrm{L}$, the sensitivity, specificity, positive predictive value (PPV), and negative predictive value (NPV) was $87.5 \%, 62.8 \%, 16.7 \%$, and $98.3 \%$, respectively. With the threshold value of D-dimer set at $0.82 \mu \mathrm{g} / \mathrm{mL}$,

Table 1 Patient characteristics

\begin{tabular}{llll}
\hline & Group 1 $(n=8)$ & Group 2 $(n=40)$ & Group 3 $(n=54)$ \\
\hline Age (year) & $53.3 \pm 14.9$ & $51.4 \pm 17.5$ & $56.5 \pm 14.8$ \\
Gender & & & 0.333 \\
$\quad$ Male & $5(62.5 \%)$ & $13(32.5 \%)$ & $28(51.9 \%)$ \\
$\quad$ Female & $3(37.5 \%)$ & $27(67.5 \%)$ & $26(48.1 \%)$ \\
BMl $\left(\mathrm{kg} / \mathrm{m}^{2}\right)$ & $24.6 \pm 4.0$ & $25.3 \pm 3.5$ & $23.5 \pm 3.6$ \\
\hline
\end{tabular}



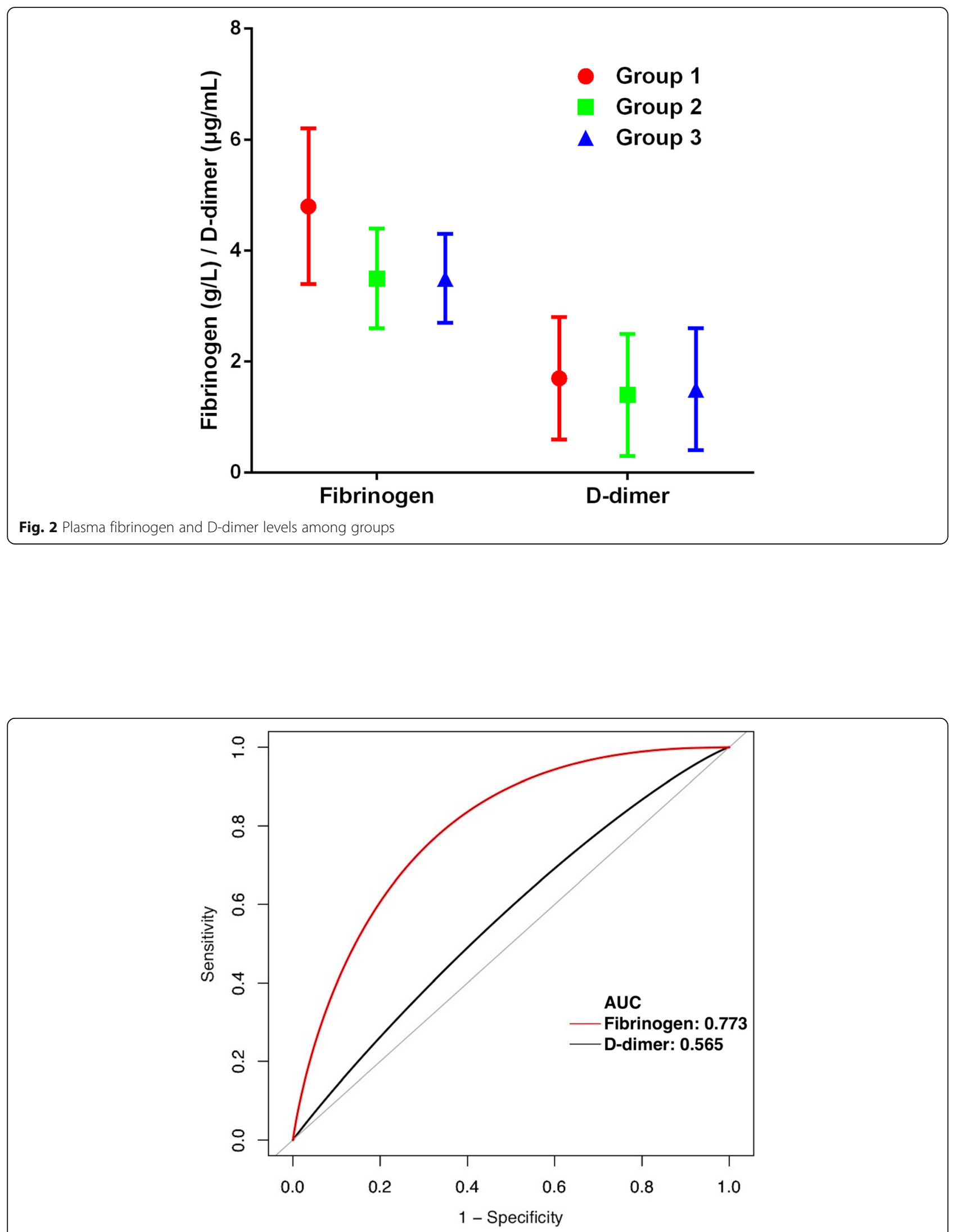

Fig. 3 Receiver operating characteristic (ROC) curves for fibrinogen and D-dimer in predicting persistent infection at the time of reimplantation 
Table 2 Values of fibrinogen and D-dimer in predicting persistent infection before reimplantation

\begin{tabular}{|c|c|c|c|c|c|c|c|}
\hline & AUC & $95 \% \mathrm{Cl}$ & Threshold & Sensitivity & Specificity & Positive predictive value & Negative predictive value \\
\hline Fibrinogen & 0.773 & $0.569-0.905$ & $3.61 \mathrm{~g} / \mathrm{L}$ & $87.5 \%$ & $62.8 \%$ & $16.7 \%$ & $98.3 \%$ \\
\hline D-dimer & 0.565 & $0.329-0.777$ & $0.82 \mu \mathrm{g} / \mathrm{mL}$ & $83.3 \%$ & $41.9 \%$ & $21.7 \%$ & $92.9 \%$ \\
\hline \multicolumn{8}{|c|}{ Sensitivity analysis } \\
\hline Fibrinogen & 0.778 & $0.558-0.917$ & $3.61 \mathrm{~g} / \mathrm{L}$ & $87.5 \%$ & $63.0 \%$ & $25.9 \%$ & $97.1 \%$ \\
\hline D-dimer & 0.565 & $0.318-0.785$ & $0.82 \mu \mathrm{g} / \mathrm{mL}$ & $83.3 \%$ & $43.8 \%$ & $35.7 \%$ & $87.5 \%$ \\
\hline
\end{tabular}

$A U C$ area of under the receiver operating characteristic curve

the sensitivity, specificity, PPV, and NPV were $83.3 \%$, $41.9 \%, 21.7 \%$, and $92.9 \%$, respectively (Table 2 ).

\section{Sensitivity analysis}

In the first set of sensitivity analysis, only hips (54 cases in group 3) without any minor criteria (as per above) were considered as infection eradication at the time of reimplantation. The results of sensitivity analysis were consistent with above results; the AUC for fibrinogen (0.778; 95\% CI, 0.558-0.917) was significantly higher than that of D-dimer $(0.565$; 95\% CI, 0.318-0.785) (Table 2).

\section{Discussion}

It is critical to identify persistent infection before second-stage reimplantation. However, the optimal timing of reimplantation remains unknown due to the lack of reliable biomarkers that can monitor persistent infection. The current study revealed that plasma fibrinogen levels might predict persistence of infection at the time of reimplantation with the best threshold of $3.61 \mathrm{~g} / \mathrm{L}$. Besides, D-dimer failed to indicate benefits in predicting infection eradication.

However, when interpreting our findings, several limitations should be considered. First, it was retrospective in nature and hence was subject to the inadequacy and variability in data collection. Second, it was a single-institution study with a limited sample size and, as a result, has limited external validity. Nevertheless, bootstrap resampling ( $n=500)$, an internal validation method, was conducted in the present study to enlarge simple sizes statistically. Third, while the modified MSIS criteria were used in the current study, there has been no "gold standard" for diagnosis of persistent infection at the time of reimplantation. It is possible that cases with one minor criteria deemed to be not infected might have been infected. Nonetheless, a set of sensitivity analysis by using patients without any minor criteria as controls was performed to minimize bias. Although several studies defined persistence of infection by using treatment failure following reimplantation [5, 6, 21], it was not applied in this study as it might be not reasonable. For example, the persistence of infection before reimplantation can be eradicated during the second-stage procedure, or vulnerable patients without persistent infection at the time of reimplantation may suffer from re-infection postoperatively. Fourth, prophylactic anticoagulation following the first-stage resection arthroplasty may influence the coagulation biomarker values, such as D-dimer and fibrinogen. However, all the second-stage reimplantation was performed after more than 4 weeks of Rivaroxaban withdrawal, which had limited impact on the plasma D-dimer and fibrinogen levels. Lastly, the application of current results was limited in patients without VTE and malignancy. Lastly, it is difficult to present AUCs for ESR and CRP because they are part of the elements used in the reference standard diagnosis.

To our best knowledge, this is the first study to evaluate the predictive values of coagulation-related markers in the diagnosis of persistent infection at the time of reimplantation. Close connections between fibrinolytic activities and infection/inflammatory have been proved in the literature $[14,15,22-24]$. Fibrinogen is the precursor of fibrin that is a soluble glycoprotein weighing $340 \mathrm{kDa}$. It is composed of three polypeptide chains called $\mathrm{A} \alpha$, $\mathrm{B} \beta$, and $\gamma$ and is synthesized in the liver [15]. While it has been well known for its crucial role in the coagulation cascade, fibrinogen also plays a key role in activating and mediating the inflammation process $[14,15]$. A most recent study by Klim et al. has suggested fibrinogen may be promising in the diagnosis of PJI with a sensitivity of 0.90 and a specificity of 0.34 [16]. However, further studies are required to validate the value of fibrinogen in the diagnosis of PJI.

Additionally, Busso et al. have described the mechanisms that D-dimer levels were elevated in patients with rheumatoid arthritis [25]. Currently, a retrospective study indicated D-dimer outperformed both serum ESR and CRP in the diagnosis of PJI, with a sensitivity of $89 \%$ and a specificity of 93\% [17]. Furthermore, they described five patients with raised D-dimer before reimplantation and two of them had a positive culture during reimplantation and then experienced failure due to infection postoperatively. However, serum ESR and CRP levels were normal in these two patients; hence, they speculated that D-dimer might be also valuable in predicting persistent infection before second-stage reimplantation. Unfortunately, the current study failed to identify the benefits of D-dimer in predicting persistent infection before reimplantation. The rationale may be a 
larger sample size used and different criteria for persistent infection in the present study.

Several studies have suggested classical markers used in the diagnosis of PJI, including serum ESR, CRP, synovial WBC count, and PMN\%, performed limited values for assessment of persistent infection before reimplantation [79]. Newman et al. suggested that a high incidence of "dry tap" in patients with antibiotic cement spacers of the hip. Moreover, they indicated aspirations performed with saline lavage were not reliable in predicting persistent infection [7]. Currently, a single-institution study by Kheir et al. revealed that a positive leukocyte esterase (LE) strip test (2+) might be indicative of persistence of infection and resulted in a higher rate of subsequent failure [5]. The AUC of LE they reported was only 0.632 , which was much lower than that of plasma fibrinogen (0.773) in the current study. However, this dissimilarity may be due to the different definitions for persistent infection and cohorts used.

\section{Conclusions}

In conclusion, the current study reveals that the plasma fibrinogen may be a promising biomarker in predicting persistent infection before reimplantation. However, D-dimer may have limited benefit in predicting infection eradication. Further prospective studies with larger cohorts are required to validate predictive values and optimal thresholds of coagulation-related markers.

\begin{abstract}
Abbreviations
AUC: The area under the ROC curve; BMI: Body mass index; CRP: C-reactive protein; ESR: Erythrocyte sedimentation rate; LE: Leukocyte esterase: MSIS: Musculoskeletal Infection Society; NPV: Negative predictive value; PJl: Periprosthetic joint infection; PMN\%: Percentage of polymorphonuclear cells; PPV: Positive predictive value; ROC: Receiver operating characteristic; VTE: Venous thrombus embolism; WBC: White blood cell
\end{abstract}

\section{Acknowledgements}

The authors would like to thank all staff from the participating departments and clinics.

\section{Funding}

Not applicable.

\section{Availability of data and materials}

We do not wish to share our data, because some of the patient's data regarding individual privacy, and according to the policy of our hospital, the data could not be shared with others without permission.

\section{Authors' contributions}

CX and PFQ drafted the manuscript. CX, PFQ, and RL performed data collection and data analysis. CX, PFQ, WC, and JYC conceived of the study, participated in the design of the study, performed data interpretation, and participated in coordination. All authors read and approved the final manuscript.

\section{Ethics approval and consent to participate}

This study was approved by the Ethics Committee of the General Hospital of People's Liberation Army and in accordance with the standards of the National Research Council. Written informed consent was obtained from all participants.

\section{Consent for publication}

Not applicable.

\section{Competing interests}

The authors declare that they have no competing interests.

\section{Publisher's Note}

Springer Nature remains neutral with regard to jurisdictional claims in published maps and institutional affiliations.

Received: 21 February 2019 Accepted: 30 April 2019

Published online: 14 May 2019

\section{References}

1. Mortazavi SMJ, Vegari D, Ho A, Zmistowski B, Parvizi J. Two-stage exchange arthroplasty for infected total knee arthroplasty: predictors of failure. Clin Orthop Relat Res. 2011:469:3049-54.

2. Sherrell JC, Fehring TK, Odum S, Hansen E, Zmistowski B, Dennos A, et al. The Chitranjan Ranawat Award: fate of two-stage reimplantation after failed irrigation and débridement for periprosthetic knee infection. Clin Orthop Relat Res. 2011;469:18-25.

3. Tan TL, Goswami K, Fillingham YA, Shohat N, Rondon AJ, Parvizi J. Defining treatment success after 2-stage exchange arthroplasty for periprosthetic joint infection. J Arthroplasty. 2018;33:3541-6.

4. Hoell S, Borgers L, Gosheger G, Dieckmann R, Schulz D, Gerss J, et al. Interleukin-6 in two-stage revision arthroplasty: what is the threshold value to exclude persistent infection before re-implanatation? The Bone \& Joint J. 2015;97-B:71-5.

5. Kheir MM, Ackerman CT, Tan TL, Benazzo A, Tischler EH, Parvizi J. Leukocyte esterase strip test can predict subsequent failure following reimplantation in patients with periprosthetic joint infection. The Journal of Arthroplasty. 2017;32:1976-9.

6. Zmistowski BM, Clyde CT, Ghanem ES, Gotoff JR, Deirmengian CA, Parvizi J. Utility of synovial white blood cell count and differential before reimplantation surgery. J Arthroplasty. 2017;32:2820-4.

7. Newman JM, George J, Klika AK, Hatem SF, Barsoum WK, Trevor North W, et al. What is the diagnostic accuracy of aspirations performed on hips with antibiotic cement spacers? Clin Orthop Relat Res. 2017:475:204-11.

8. Kusuma SK, Ward J, Jacofsky M, Sporer SM, Della Valle CJ. What is the role of serological testing between stages of two-stage reconstruction of the infected prosthetic knee? Clin Orthop Relat Res. 2011;469:1002-8.

9. Ghanem E, Antoci V, Pulido L, Joshi A, Hozack W, Parvizi J. The use of receiver operating characteristics analysis in determining erythrocyte sedimentation rate and C-reactive protein levels in diagnosing periprosthetic infection prior to revision total hip arthroplasty. Int J Infect Dis. 2009:13:e444-9.

10. Janz V, Bartek B, Wassilew GI, Stuhlert M, Perka CF, Winkler T. Validation of synovial aspiration in Girdlestone hips for detection of infection persistence in patients undergoing 2-stage revision total hip arthroplasty. J Arthroplasty. 2016:31:684-7.

11. George J, Kwiecien G, Klika AK, Ramanathan D, Bauer TW, Barsoum WK, et al. Are frozen sections and MSIS criteria reliable at the time of reimplantation of two-stage revision arthroplasty? Clin Orthop Relat Res. 2016;474:1619-26.

12. Cho W-S, Byun S-E, Cho W-J, Yoon Y-S, Dhurve K. Polymorphonuclear cell count on frozen section is not an absolute index of reimplantation in infected total knee arthroplasty. J Arthroplasty. 2013;28:1874-7.

13. Bauer TW, Parvizi J, Kobayashi N, Krebs V. Diagnosis of periprosthetic infection. J Bone Joint Surg Am. 2006;88:869-82.

14. Davalos D, Akassoglou K. Fibrinogen as a key regulator of inflammation in disease. Semin Immunopathol. 2012;34:43-62.

15. Jennewein C, Tran N, Paulus P, Ellinghaus P, Eble JA, Zacharowski K. Novel aspects of fibrin(ogen) fragments during inflammation. Mol Med. 2011;17:568-73.

16. Klim SM, Amerstorfer F, Gruber G, Bernhardt GA, Radl R, Leitner L, et al. Fibrinogen - A practical and cost efficient biomarker for detecting periprosthetic joint infection. Sci Rep. 2018;8:8802.

17. Shahi A, Kheir MM, Tarabichi M, Hosseinzadeh HRS, Tan TL, Parvizi J. Serum D-Dimer test is promising for the diagnosis of periprosthetic joint infection and timing of reimplantation. J Bone Joint Surg. 2017;99:1419-27.

18. Parvizi J, Gehrke T. Definition of periprosthetic joint infection. J Arthroplasty. 2014;29:1331

19. Nalls MA, McLean CY, Rick J, Eberly S, Hutten SJ, Gwinn K, et al. Diagnosis of Parkinson's disease on the basis of clinical and genetic classification: a population-based modelling study. Lancet Neurol. 2015;14:1002-9. 
20. DeLong ER, DeLong DM, Clarke-Pearson DL. Comparing the areas under two or more correlated receiver operating characteristic curves: a nonparametric approach. Biometrics. 1988;44:837-45.

21. Berbari E. CORR Insights ${ }^{\oplus}$ : synovial cytokines and the MSIS criteria are not useful for determining infection resolution after periprosthetic joint infection explantation. Clin Orthop Relat Res. 2016;474:1640-2.

22. Michelin E, Snijders D, Conte S, Dalla Via P, Tagliaferro T, Da Dalt L, et al. Procoagulant activity in children with community acquired pneumonia, pleural effusion and empyema. Pediatr Pulmonol. 2008;43:472-5.

23. Gando S. Role of fibrinolysis in sepsis. Semin Thromb Hemost. 2013;39:392-9.

24. Ribera T, Monreal L, Armengou L, Ríos J, Prades M. Synovial fluid D-dimer concentration in foals with septic joint disease. J Vet Intern Med. 2011;25:1113-7.

25. Busso N, Hamilton JA. Extravascular coagulation and the plasminogen activator/plasmin system in rheumatoid arthritis. Arthritis Rheum. 2002:46:2268-79

Ready to submit your research? Choose BMC and benefit from:

- fast, convenient online submission

- thorough peer review by experienced researchers in your field

- rapid publication on acceptance

- support for research data, including large and complex data types

- gold Open Access which fosters wider collaboration and increased citations

- maximum visibility for your research: over $100 \mathrm{M}$ website views per year

At BMC, research is always in progress.

Learn more biomedcentral.com/submissions 